\title{
Female Outperformance in Voluntary Running Persists in Dystrophin-Null and Klotho-Overexpressing Mice
}

\author{
Michael Phelps ${ }^{\mathrm{a}, *}$ and Zipora Yablonka-Reuveni ${ }^{\mathrm{b}, *}$ \\ ${ }^{a}$ Department of Animal Sciences, Washington State University, Pullman, WA, USA \\ ${ }^{\mathrm{b}}$ Department of Biological Structure, University of Washington School of Medicine, Seattle, WA, USA
}

Pre-press 10 July 2021

\begin{abstract}
.
Background: Duchenne muscular dystrophy is a degenerative muscle disease that results from impairment of the dystrophin gene. The disease causes progressive loss in muscle mass and function.

Objective: The anti-aging protein, $\alpha$-klotho, has been implicated in the regulation of muscle regeneration. We previously discovered that mice harboring reduced $\alpha$-klotho levels exhibited a decline in muscle strength and running endurance.

Method: To investigate the ability of $\alpha$-klotho to improve overall endurance in a dystrophin null murine model, we examined the voluntary wheel running performance of dystrophin-null, $\mathrm{mdx}^{4 \mathrm{cv}}$ mice overexpressing an $\alpha$-klotho transgene.

Results: As expected, compared to wild type, both male and female dystrophic mice exhibited reduced running ability that was characterized by shorter running duration and longer periods of rest between cycles of activity. While our results did not detect an improvement in running performance with $\alpha$-klotho overexpression, we identified distinct differences in the running patterns between females and males from all mouse strains analyzed (i.e., $\mathrm{mdx}^{4 \mathrm{cv}}, \mathrm{mdx}^{4 \mathrm{cv}}$ overexpressing $\alpha$-klotho, $\alpha$-klotho overexpressing, $\alpha$-klotho hypomorph, and wild type). For all strains, male mice displayed significantly reduced voluntary running ability compared to females. Further analysis of the $\mathrm{mdx}^{4 \mathrm{cv}}$ strains demonstrated that male mice ran for shorter lengths of time and took longer breaks. However, we did not identify gender-associated differences in the actual speed at which $\mathrm{mdx}^{4 \mathrm{cv}}$ mice ran.

Conclusion: Our data suggest key differences in the running capabilities of female and male mice, which are of particular relevance to studies of dystrophin-null mice.
\end{abstract}

Keywords: Running wheel, $\mathrm{mdx}, \mathrm{mdx}^{4 \mathrm{cv}}, \alpha$-klotho

\section{INTRODUCTION}

The loss of a functional dystrophin protein in Duchenne muscular dystrophy results in a gradual deterioration of muscle tissue and premature death. The sustained muscle injury associated with

${ }^{*}$ Correspondence to: Michael Phelps and Zipora YablonkaReuveni, Department of Animal Sciences, Washington State University, Pullman, WA, USA and Department of Biological Structure, University of Washington School of Medicine, Seattle, WA, USA. E-mail: reuveni@uw.edu. muscular dystrophy results in significant muscle tissue fibrosis [1]. Increased fibrosis has also been implicated in age-associated changes in skeletal muscle [2]. The anti-aging protein $\alpha$-klotho (AKA klotho, the term used below throughout the manuscript) has a well-established role in protecting against renal fibrosis [3] and loss of klotho function was shown to increase muscle fibrosis in aging and dystrophic mouse models $[4,5]$. Klotho is primarily expressed in the kidneys but has also been shown to be expressed in muscle tissue at a low level with increased expression 
in response to injury [4-6]. Klotho expression is reduced in dystrophic mdx mice (i.e., mice lacking functional dystrophin in the muscle tissue and considered dystrophin-null) and in aged mice, suggesting that loss of muscle function correlates with decreases in klotho expression [4]. Evidence suggests that the reduction in klotho expression in skeletal muscle with age is the result of epigenetic silencing of the klotho gene $[4,5]$. In addition to localized klotho expression, klotho is also known to have a secretory form that is hypothesized to be responsible for many of the more global functions attributed to the klotho protein [7]. The protective function of klotho in skeletal muscle is potentially due to the ability of klotho to improve muscle stem cell function [4]. Loss of function mutations in the klotho gene lead to a reduction in muscle stem cell numbers in mice and proliferation in culture [6]. In addition to improving muscle stem cell function, it has been suggested that klotho overexpression may be able to improve muscle tissue aging and regeneration and reduce disease severity in dystrophic $(\mathrm{mdx})$ mouse models through a yet to be identified mechanism $[4,5]$.

Despite growing evidence suggesting that klotho expression can improve muscle function in dystrophic mice, more data is needed on the effect of klotho on overall muscle physiology and function. Klotho null mice exhibit a significant reduction in voluntary wheel running performance and grip strength [8]. However, overexpression of klotho in wild type mice did not improve overall strength or running capabilities [8] supporting the idea that klotho function in muscle tissue is activated in response to muscle injury. Since klotho expression is significantly reduced in dystrophic muscle we have hypothesized that overexpression of klotho can improve running endurance capabilities in dystrophin-null mice.

While muscle function and performance have at times been reported without a reference to the gender analyzed or with the statement that equal numbers of males and females were used per treatment, a growing body of research highlights strong differences in the running performance or activity level of male and female mice [1, 9-13]. Physiological differences between males and females can compound experimental results by averaging the natural variation found between genders [14, 15]. Mdx mice have been shown to have reduced running capabilities that are shorter in duration than healthy mice [16]. However, the extent of gender difference in running performance in $\mathrm{mdx}$ mice is not well studied.
Different approaches have been used to study physical activity/endurance in mdx mice, including swimming, forced treadmill running, and voluntary wheel running $[17,18]$. Here, we studied mouse activity using voluntary wheel running, an experimental model where mice are running at will during their active night hours and are acclimated to their steady cage environment for the duration of the experiment $[8,16,19]$. To examine the effect of klotho on overall running capabilities in dystrophin-null mice, we measured voluntary wheel running ability in $\mathrm{mdx}^{4 \mathrm{cv}}$ mice overexpressing klotho, compared to $\mathrm{mdx}^{4 \mathrm{cv}}$ and wild type mice. $M d x^{4 c v}$ mice differ from $\mathrm{mdx}$ mice in the specific mutation in the dystrophin gene and display a reduced spontaneous appearance of dystrophin positive myofibers compared to $\mathrm{mdx}$ mice [20-22]. While klotho overexpression did not influence overall running ability in dystrophic mice, we identified significant differences in running capability between female and male mice that highlight the need for careful gender consideration of running wheel experimental data.

\section{MATERIAL AND METHODS}

\section{Mice}

All mice used in the current study were from colonies maintained by us on a C57BL/6 strain background for long term at the University of Washington. Animal procedures used in the research were approved by the University of Washington Institutional Animal Care and Use Committee. Mice were housed in a specific pathogen-free facility under 12/12-h light/dark cycle and were fed ad libitum Lab Diet 5053. The initial klotho hypomorphic (Kl-null) and transgenic klotho-overexpressing mice $(K l$; transgene driven by the Ef $1 \alpha$ promoter) were generously provided by Dr. Makoto Kuroo (UT Southwestern Medical Center). Both klotho hypomorphic and overexpressing mouse strains were maintained by us as homozygous lines for analysis as we previously described [8]. The initial $\mathrm{mdx}^{4 \mathrm{cv}}$ mice were kindly provided by Dr. Jeffery Chamberlain (University of Washington School of Medicine). $\mathrm{Mdx}^{4 \mathrm{cv}}$ mice overexpressing klotho were created by first crossing homozygous klotho transgenic $(K l)$ male and homozygous $\mathrm{mdx}^{4 \mathrm{cv}}$ female mice. Since dystrophin is an X-chromosome linked gene, all males resulting from this first breeding step were $\mathrm{mdx}^{4 \mathrm{cv}}$ mice harboring a hemizygous klotho transgene. These males were then back-crossed to homozygous $\mathrm{mdx}^{4 \mathrm{cv}}$ females to generate experimen- 
tal male and female $\mathrm{mdx}^{4 \mathrm{cv}}$ mice with or without the klotho transgene. All $\mathrm{mdx}^{4 \mathrm{cv}}$ klotho transgenic mice were hemizygous for the transgene through this breeding scheme; all females resulting from this breeding scheme were homozygous for the $\mathrm{mdx}$ mutation (i.e., both X-chromosomes affected). Genotyping the Ef1 $\alpha$ driven klotho transgene was performed by PCR amplification of the transgene, as previously described [8]. Genotyping the $\mathrm{mdx}^{4 \mathrm{cv}}$ allele was verified through sequencing [23]. All mice were genotyped at weaning and again prior to experimentation. To validate that the klotho transgene was overexpressed in our transgenic mice we confirmed transgene expression in representative age matched mice just prior to experimentation, using reverse transcription quantitative PCR (RT-qPCR) to quantify the klotho transcript level in the diaphragm, tibialis anterior (TA), and kidney (Supplemental Figure S1). The primer sets and RT-PCR protocols used for gene expression analysis of the klotho transgene are described in detail in Phelps et. al., 2013. The dramatically higher klotho expression in muscles of mice harboring the klotho transgene compared to endogenous expression (Figure S1) is in agreement with our previously published study.

\section{Voluntary running analysis}

We performed the study using equipment from the In Vivo Services (IVS) Core in the Department of Comparative Medicine, University of Washington School of Medicine. Low-profile $15.5 \mathrm{~cm}$ diameter wireless running wheels (Med Associates, Inc.) were used to compare continuous voluntary running activity between mice as previously described by us [8]. Mice were isolated into individual cages and acclimated to cage conditions containing a locked running wheel for 3 days prior to recording data. Voluntary wheel running activity was measured simultaneously across all mouse strains for 8 consecutive days. The following numbers of animals were analyzed for each experimental group ( $\mathrm{Kl}$-null: $n=6(3 \mathrm{~F}, 3 \mathrm{M}), K l: n=12$ (9F, 3M), WT: $n=13$ (6F, $7 \mathrm{M}), \mathrm{mdx}^{4 \mathrm{cv}}: n=15(5 \mathrm{~F}, 10 \mathrm{M})$, and $K l-\mathrm{mdx}^{4 \mathrm{cv}}$ : $n=16(8 \mathrm{~F}, 8 \mathrm{M})$; all mice were $8-11$ months of age). Wireless running wheels record the number of revolutions completed every minute throughout the study. Running activity recorded for only one minute was not counted in the analysis as it could represent accidental spinning of the wheel during normal cage activity. From these data we calculated the following experimental parameters.
A. The total number of revolutions completed by each mouse over the 8-day experiment was calculated directly from the raw running wheel data.

B. Running speed was calculated by taking the average number of revolutions performed per minute for each mouse while running.

C. Actograms were created by plotting the revolutions recorded every minute into a single graph using Excel sparklines. As we previously detailed [8], The actograms reveal the overall trends in running activity throughout the day in relation to the light/dark cycle.

D. To calculate the number of running intervals (periods of running activity) for each mouse the initiation and cessation of running activity was identified from the voluntary running wheel data. This information was used to determine the number of individual running intervals regardless of the duration of those intervals.

E. The length of each running interval was calculated by converting the running wheel data into binary data. This binary data was used to determine the percentage of time spent running by dividing the total active running time by the total experimental time.

Percent Time Running =

$\underline{\text { No. of Running Intervals } \times \text { Interval Duration }}$ Total Time (8 days)

F. The duration of each running and resting interval was then calculated from this binary data by summing the number of minutes for each running and resting interval. The length of these running and resting intervals was then sorted into one and five minute bins, to determine the length distribution of running and resting intervals.

G. The cumulative percentage of running interval lengths was calculated by summing the number of intervals in each bin, starting with the 2 minute bin and dividing by the total number of intervals ran throughout the experiment. Cumulative resting intervals were not calculated due to the fact that long periods of sustained resting occur when the animals slept.

\section{Statistics}

When comparing the voluntary wheel running results between males and females within each strain 
a $t$-test was used and differences were considered significant at $p<0.05$ (black asterisks in figures). When comparing the same gender across mouse strains, the data was first analyzed using a two-way ANOVA, followed by post hoc analysis with individual $t$-tests; the significance level was adjusted with the Bonferroni correction $p<0.017$ (gray asterisk in figures), [8]. All running wheel graphs present the average experimental values \pm SEM.

\section{RESULTS}

\section{Klotho overexpression failed to influence running ability in a muscular dystrophy mouse model}

As detailed in the Introduction, the decreased muscle function in klotho deficient mice that our lab and others have demonstrated along with the well documented function of klotho in renal fibrosis led us to hypothesize that overexpression of klotho could be used to improve muscle function in dystrophin mutant mice. To test this hypothesis we established klotho $(K l)$ overexpressing transgenic $\mathrm{mdx}^{4 \mathrm{cv}}$ mice $\left(K l-\mathrm{mdx}^{4 \mathrm{cv}}\right)$ and examined their overall physical activity and endurance with voluntary wheel running. $\mathrm{Mdx}^{4 \mathrm{cv}}$ mice were created by chemically induced mutagenesis and lack a full-length functional dystrophin protein due to a $\mathrm{C}$ to $\mathrm{T}$ transition mutation in the dystrophin gene that leads to a premature stop codon and truncation of the protein [24]. This leaves the mice functionally null for dystrophin in skeletal muscle. While smaller dystrophin transcripts are observed in non-muscle tissues they are not expressed in skeletal muscle $[22,25] . \mathrm{Mdx}^{4 \mathrm{cv}}$ mice are also 10 -fold less susceptible to random activation of fulllength dystrophin in myofibers (i.e., "revertants") compared to what has been observed in standard mdx mice [20-22]. Because of this, the $\mathrm{mdx}^{4 \mathrm{cv}}$ strain has been preferred by various laboratories when using dystrophin-null mice as a model for Duchenne muscular dystrophy.

To verify the presence of elevated levels of klotho expression in our transgenic klotho overexpressing $\mathrm{mdx}^{4 \mathrm{cv}}$ mouse model we analyzed klotho gene expression in the diaphragm, tibialis anterior muscle (TA), and in the kidney, the dominant location for natural klotho expression (Supplemental Figure S1). We confirmed that transgenic $\mathrm{mdx}^{4 \mathrm{cv}}$ animals of both genders expressed highly elevated levels of klotho expression in the muscle tissues, which is in agreement with our previous studies [8]. Additional klotho expression in kidneys of klotho transgenic mice was not detected above the already very high endogenous klotho expression.

Voluntary wheel running is a sensitive method for evaluating endurance and activity of mice, which is associated with overall musculoskeletal system function [8]. Our voluntary wheel running experiment measured the number of wheel rotations every minute over an 8-day period of time. The performance of

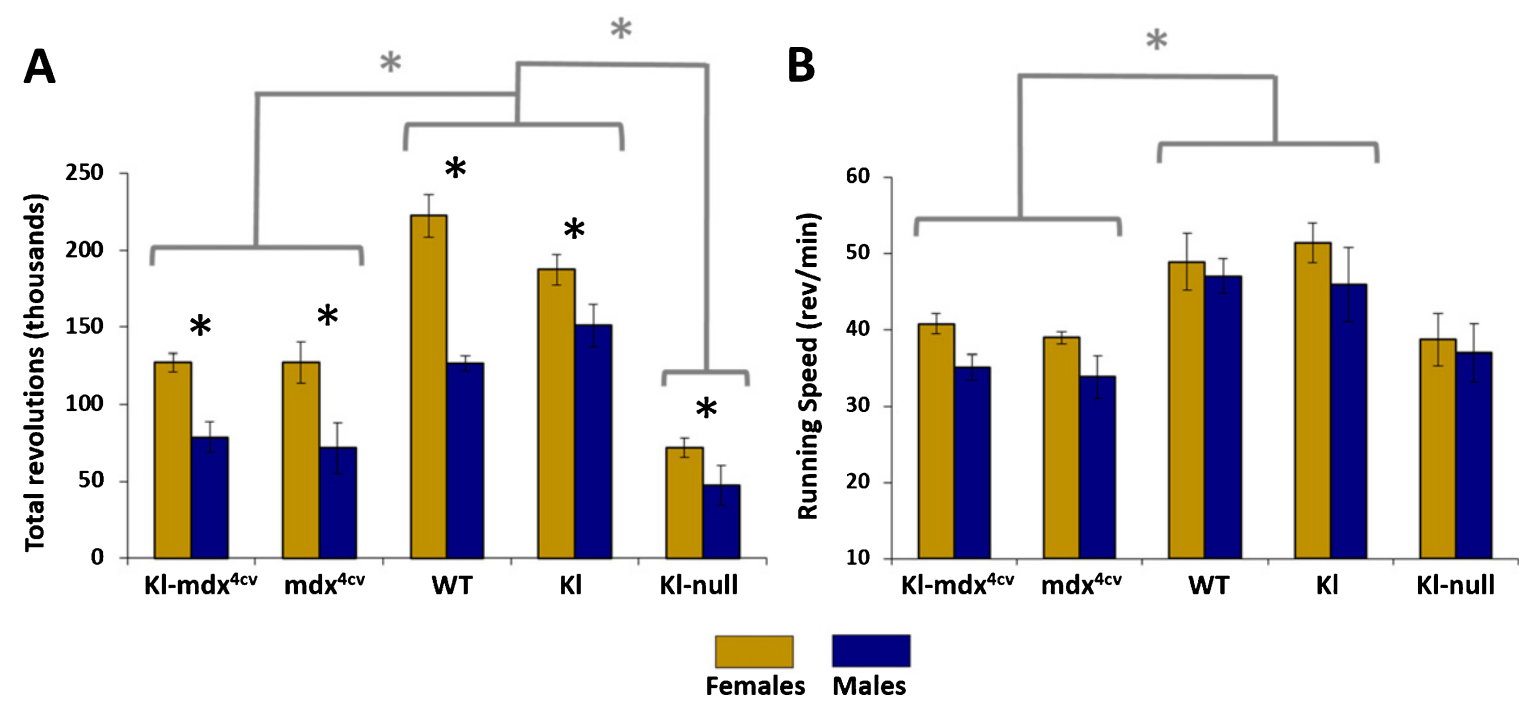

Fig. 1. Total running distance and speed. (A) The average total number of revolutions performed over 8 consecutive days across all mouse strains for both female (gold) and male (blue) mice. (B) The average speed of mice while running as determined by the number of revolutions performed per minute. Black * represents statistical differences between males and females within same mouse strain $(p<0.05)$; gray * and brackets represent statistical differences across identified groups of mouse strains for both males and females, analyzed independently per each gender $(p<0.017)$. 
both male and female $K l-\mathrm{mdx}^{4 \mathrm{cv}}$ mice was compared to $\mathrm{mdx}^{4 \mathrm{cv}}, K l$-null, $K l$-transgenic $(K l)$, and wild type C57B6 mice (WT) to determine the impact of performance between strains and genders. We did not identify a significant difference in the total distance ran between $\mathrm{Kl}$ - $\mathrm{mdx}^{4 \mathrm{cv}}$ and $\mathrm{mdx}^{4 \mathrm{cv}}$ mice over the 8-day period (Fig. 1A). There was however, a significant reduction in total running performance in $K l$ - $\mathrm{mdx}^{4 \mathrm{cv}}$ and $\mathrm{mdx}^{4 \mathrm{cv}}$ compared to wild type and $K l$ transgenic controls (Fig. 1A). Klotho-null mice exhibited the worst running performance of all experimental groups, which is in agreement with our previous studies investigating voluntary wheel running in these mice (Fig. 1A) [8].

\section{Female mice run longer distances than their male counterparts}

While some variation in the running abilities of male and female mice were expected, we did not anticipate the extent to which the genders differed based off of the overall running distance (i.e., total revolutions). Females of all of the groups analyzed showed significantly increased running capabilities compared to males (Fig. 1A). The better running performance of females could not be accounted for by differences in the average running speed (revolutions/min) of the mice since whereas female mice tended to run faster, the pace was not significantly different from that of male mice for most treatment groups (Fig. 1B). Notably, the running speed of $K l$ $\mathrm{mdx}^{4 \mathrm{cv}}$ and $\mathrm{mdx}^{4 \mathrm{cv}}$ was still reduced compared wild type mice but not to the extent observed when analyzing overall running distance (i.e., total revolutions; Fig. 1A). No difference was observed in total revolutions between wild type and klotho-overexpressing mice (Fig. 1A).

\section{Performance actograms reveal reduced engagement in running in male compared to female mice}

To investigate the cause of reduced running performance in both male and female $\mathrm{mdx}^{4 \mathrm{cv}}$ mice we analyzed the overall running profile of the mice, over 8 consecutive days, using performance actograms. Since we did not observe a difference in running

\section{Females}

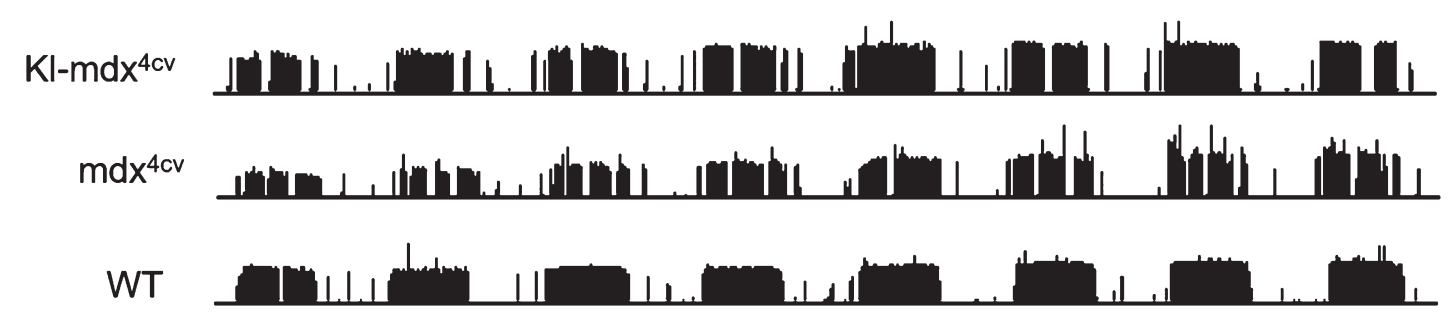

\section{Males}

Kட-maxaco

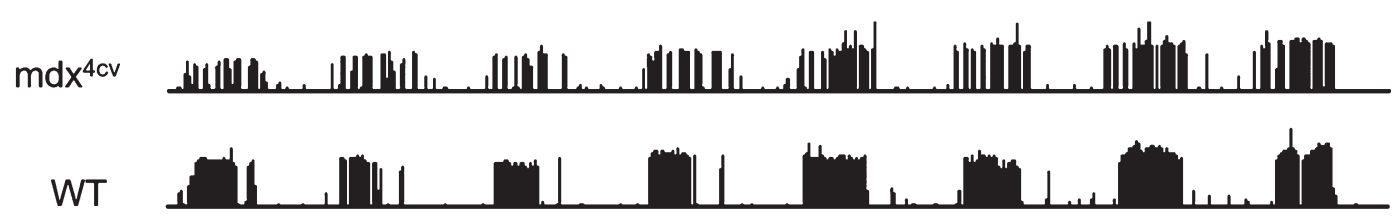

12-hr dark cycle

\begin{tabular}{lllllll}
\hline Night & Night & Night & Night & Night & Night & Night
\end{tabular}

Fig. 2. Performance actograms comparing running activity of representative $\mathrm{Kl}-\mathrm{mdx}^{4 \mathrm{cv}}$, $\mathrm{mdx}^{4 \mathrm{cv}}$ and wild type (WT) mice over 8 days of voluntary wheel running. Day and night periods are represented by horizontal light and dark bars at the bottom of the actograms. The height of each actogram bar represents the revolutions traveled during that minute of the experiment. 
performance between WT and $K l$ transgenic mice we focused the remainder of our study on the running performance of $K l$ overexpressing $\mathrm{mdx}^{4 \mathrm{cv}}$ mice $\left(\mathrm{Kl}-\mathrm{mdx}^{4 \mathrm{cv}}\right)$ compared to $\mathrm{mdx}^{4 \mathrm{cv}}$, and wild type mice (Fig. 2). Actograms highlight running trends throughout the study to show differences, if any, in the pattern of running activity between mice $[8,26]$. The height of each vertical bar represents the number of revolutions that were performed for a single minute and this is plotted over the entire 8 day experiment to show the precise periods of activity (vertical bars) and inactivity (no vertical bars, as established in numerous studies and demonstrated here, extended periods of mouse inactivity are typically associated with day time). The actograms showed significant fragmentation of the active running intervals in $K l$-mdx ${ }^{4 \mathrm{cv}}$ and $\mathrm{mdx}^{4 \mathrm{cv}}$ mice compared to wild type mice. This was represented by periods of inactivity or breaks in the nocturnal cycles of activity, which did not occur as frequently in wild type mice (Fig. 2). Interestingly, fragmented running cycles were more pronounced in male mice of all groups (Fig. 2).

To determine if the reduced running performance observed in males was due to a decrease in the number of running intervals (i.e., a running interval is a single sustained stretch of running activity without taking a break), we quantified the total number of running intervals ran by $\mathrm{Kl}$ - $\mathrm{mdx}^{4 \mathrm{cv}}, \mathrm{mdx}^{4 \mathrm{cv}}$, wild type mice throughout the study. We did not detect any difference in the number of intervals ran between these groups when analyzed within genders (Fig. 3A).
Male mice of all strains however performed significantly less running intervals than females (Fig. 3A). This contributed to the observed increase in the overall percentage of time spent running for female mice compared to male mice in all treatment groups (Fig. 3B). While there was no difference in the percentage of time spent active in male mice from all 3 groups analyzed, female $K l-\mathrm{mdx}^{4 \mathrm{cv}}$ and $\mathrm{mdx}^{4 \mathrm{cv}}$ mice spent significantly less time running then wild type females (Fig. 3B). Overall, our data suggest that males from all mouse strains spend less time running on the wheel than females and that this is due in part to a reduction in the total number of running intervals.

Our data led us to search for a possible explanation as to why female $K l-\mathrm{mdx}^{4 \mathrm{cv}}$ and $\mathrm{mdx}^{4 \mathrm{cv}}$ mice spent a lower percentage of their time running compared to wild type mice (Fig. 3B) despite showing no difference in the number of running intervals performed throughout the experiment. To address this subject further we analyzed the duration of the running intervals (i.e., running length) as well as resting intervals (i.e., resting length) as a frequency distribution across the treatment groups. Voluntary running wheel experiments measure a running event by counting the number of rotations of the wheel every minute of exercise. The duration of each running interval was quantified from this data by analyzing how long the mice spent on the wheel during each interval before taking a break and how much time was spent resting between running intervals. Wheel rotations were only counted if they were active for equal to or longer

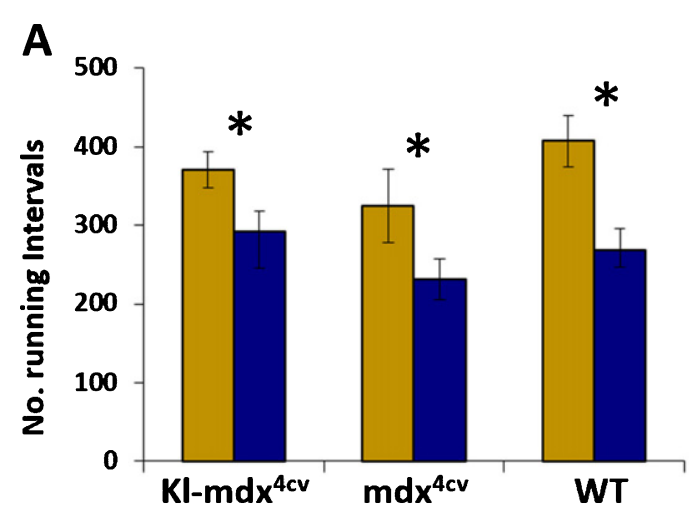

B

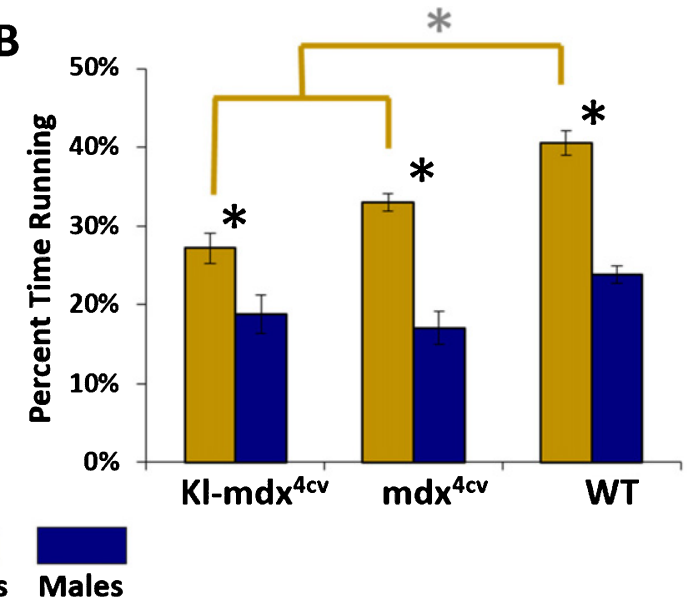

Fig. 3. Total running intervals and the percentage of time spent running. (A) The average number of running intervals ran across Kl-mdx ${ }^{4 c v}$, $\mathrm{mdx}^{4 \mathrm{cv}}$, and wild type (WT) mice. Female (gold) and male mice (blue) for all strains are identified. (B) The percentage of time the mice spent actively running throughout the experiment. Black * represents statistical differences between males and females within each strain $(p<0.05)$; gray ${ }^{*}$ and gold brackets represent statistical differences between Kl-mdx ${ }^{4 c v}$ and mdx ${ }^{4 c v}$ females versus WT females $(p<0.017)$. 


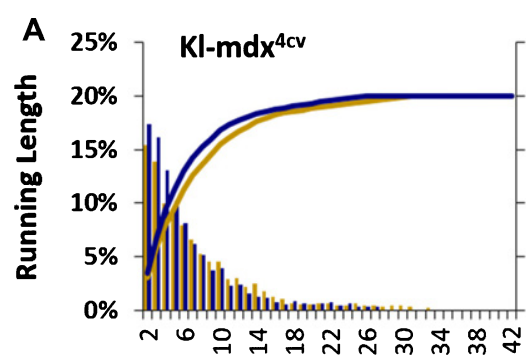

Running Length (min)

Median: Female 5.20, Male 4.33

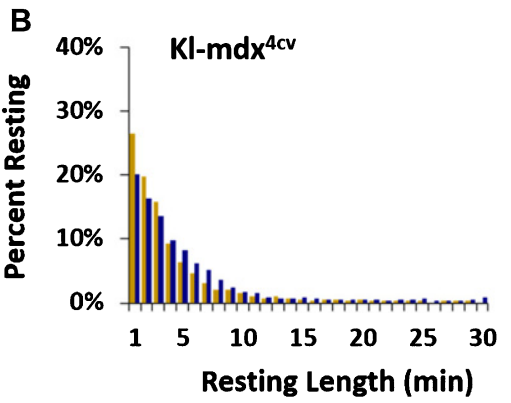

Median: Female 2.23, Male 3.00

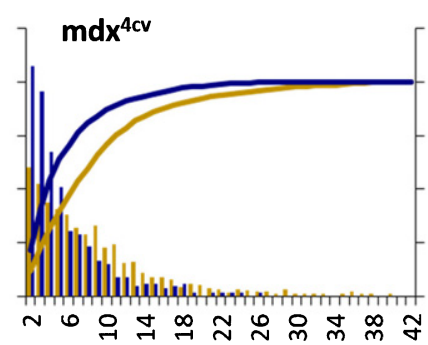

Running Length (min)

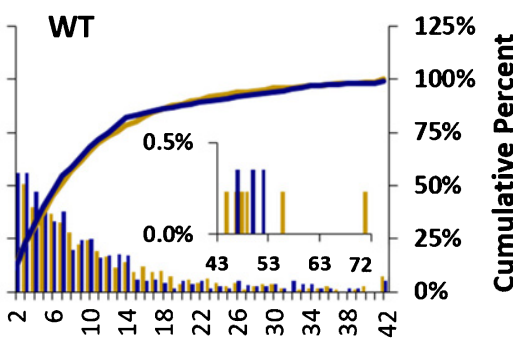

Running Length (min)

Median: Female 6.44, Male 3.70

Median: Female 6.83, Male 6.41
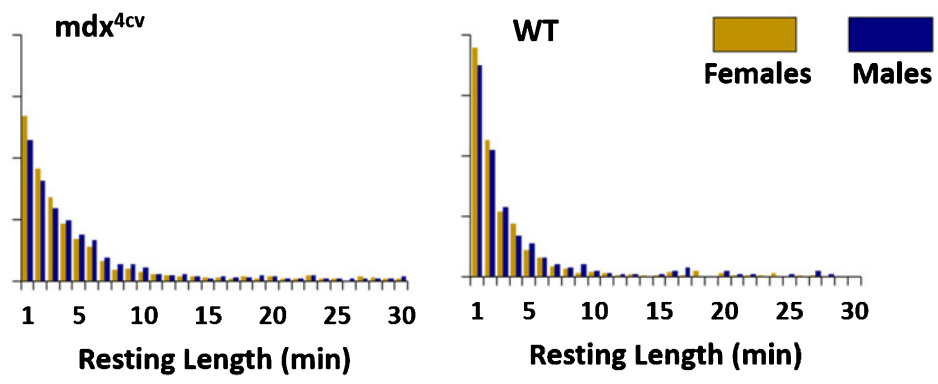

Median: Female 2.35, Male 2.89

Fig. 4. Duration of running and resting intervals. (A) The number of all one minute running interval lengths performed during the study presented as a frequency distribution along with the cumulative percentage of time spent running shown by the line graphs. An insert was placed in the wild type (WT) graph to highlight intervals ran for longer than $42 \mathrm{~min}$ without a break. These long running intervals were only observed in the WT group. (B) The length of resting intervals between cycles of active running. Females (gold) and males (blue) are shown across $K l-\mathrm{mdx}^{4 \mathrm{cv}}, \mathrm{mdx}^{4 \mathrm{cv}}$, and WT mice.

than 2 minutes to avoid artificial rotations caused by accidental movement of the wheel. We then grouped the running and resting intervals (active and resting states) into bins (1 minute) based off of their duration and plotted their frequency distribution (Fig. 4). We also calculated the cumulative rate of change between each of the $1 \mathrm{~min}$ bins for running length and plotted it as a percentage of the total running intervals for both male and female mice (Fig. 4). This analysis highlighted key differences between male and female $K l-\mathrm{mdx}^{4 \mathrm{cv}}$ and $\mathrm{mdx}^{4 \mathrm{cv}}$ mice over time that was not present in wild type mice. Male $K l-\mathrm{mdx}^{4 \mathrm{cv}}$ and $\mathrm{mdx}^{4 \mathrm{cv}}$ mice showed a trend toward running for a shorter time interval than females (Fig. 4A).

To quantify the trends that we observed in the running and resting frequency distributions we regrouped the data into $5 \mathrm{~min}$ bins and compared the percentage of total running time represented by specific interval lengths. We analyzed running lengths from $2-5 \mathrm{~min}$ and $11-15 \mathrm{~min}$ long as these intervals were characteristic of opposing ends of the frequency distribution (short and long interval durations). Through this analysis we discovered that
$K l$ - $\mathrm{ddx}^{4 \mathrm{cv}}$ and $\mathrm{mdx}^{4 \mathrm{cv}}$ mice were active for shorter lengths of time when compared to wild type males and $K l-\mathrm{mdx}^{4 \mathrm{cv}}$ and $\mathrm{mdx}^{4 \mathrm{cv}}$ females (Fig. 5A). Surprisingly, this trend was not observed between female $K l-\mathrm{mdx}^{4 \mathrm{cv}}, \mathrm{mdx}^{4 \mathrm{cv}}$, and wild type mice, which did not differ in the length of time they spent actively running (Fig. 5A). We did not observe a statistical difference for the length time spent resting between female and male mice from any of the treatment groups (Fig. 5B). The $K l-\mathrm{mdx}^{4 \mathrm{cv}}$ and $\mathrm{mdx}^{4 \mathrm{cv}}$ mice did however exhibit a significant shift toward longer resting intervals between cycles of active running when compared to wild type mice (Fig. 5B). Klotho overexpression did not influence the length of time the mice spent in active running or resting (Fig. 5).

\section{DISCUSSION}

In this study we examined the effect of klotho transgenic overexpression on voluntary wheel running in dystrophin-null $\left(\mathrm{mdx}^{4 \mathrm{cv}}\right)$ mice, investigating also the performance of $\mathrm{mdx}^{4 \mathrm{cv}}$ mice versus wild type 

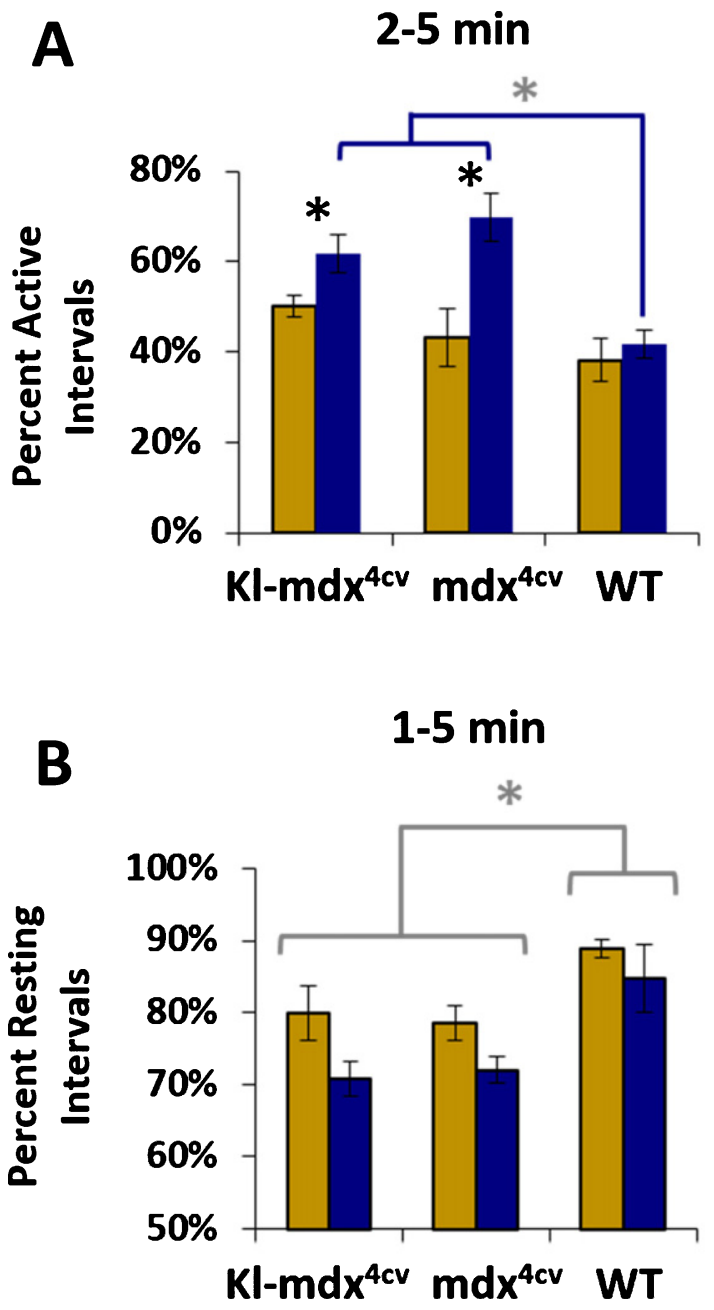

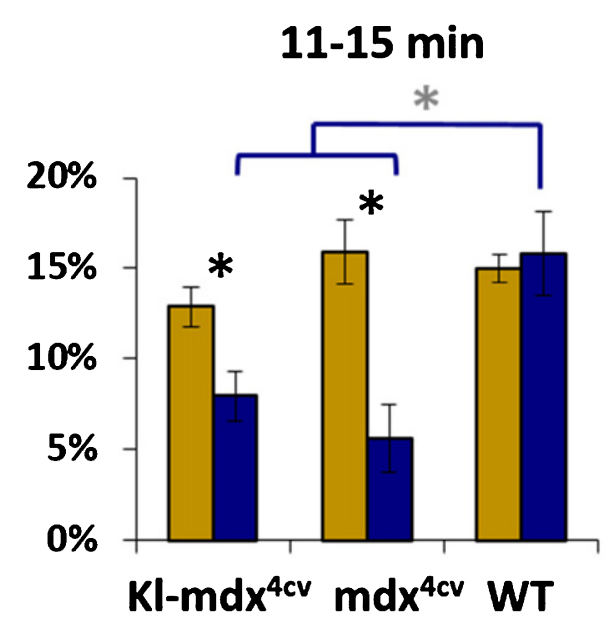

11-15 $\mathrm{min}$

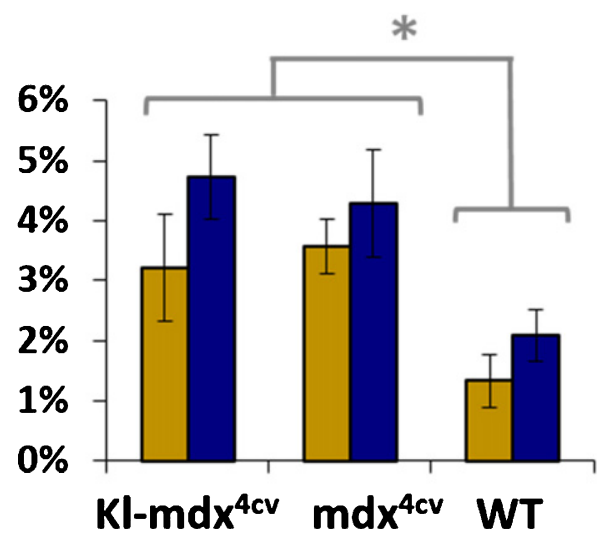

\section{Females Males}

Fig. 5. Comparison of short and long intervals for active running or resting. (A) Comparison of the 2-5 min and 11-15 minute long running intervals. (B) Analysis of the length of resting intervals between 1-5 min and 11-15 min in length. Females (gold) and males (blue) are identified along with mouse strains $\left(K l-\mathrm{mdx}^{4 \mathrm{cv}}, \mathrm{mdx}^{4 \mathrm{cv}}\right.$, and wild type (WT)). Black ${ }^{*}$ are representative of statistical differences between males and females within each strain $(p<0.05)$; gray * and blue brackets (panel A) represent statistical differences between $K l$-mdx ${ }^{4 \mathrm{cv}}$ and $\mathrm{mdx}^{4 \mathrm{cv}}$ males versus WT males $(p<0.017)$; gray ${ }^{*}$ and brackets (panel B) represent statistical differences between $K l$-mdx ${ }^{4 \mathrm{cv}}$ and $\mathrm{mdx}^{4 \mathrm{cv}}$ mice versus WT mice for both males and females analyzed independently per each gender $(p<0.017)$.

controls, with special attention to female versus male performance.

Overexpression of klotho in transgenic mice has been implicated in improving organ function during disease in several tissue groups such as kidney, brain, and cardiac muscle [27-29]. While klotho has been shown to exhibit a wide range of functions throughout the body, one of its primary roles is in regulating calcium homeostasis through an FGF23 dependent mechanism [30]. Two klotho overexpressing transgenic mice have been reported to extend lifespan in mice and improve tissue aging [31]. Expression of klotho in the EFmKL46 transgenic mouse strain (used in the present study) is driven by the Ef $1 \alpha$ promoter and has been shown to produce high levels of klotho expression in skeletal muscle in addition to systemic expression $[8,31]$. We previously demonstrated a correlation between reduced klotho expression and skeletal muscle strength where grip strength and running endurance were compared along klotho hypomorphic, klotho-overexpressing and wild type mice (study was limited to female sub- 
jects). Klotho hypomorphic mice were significantly weaker, and displayed strength approximately 50\% less than both klotho-overexpressing and wild type controls (there was no difference between klothooverexpressing and wild type controls). Interestingly, klotho hypomorphic mice in the present study, as in our previous work, ran on a running wheel at the same speed as the other two groups, but had an endurance capacity approximately $60 \%$ less than that of the control groups.

Different from previous work detecting a positive effect of klotho when examining treadmill running in klotho-overexpressing mdx mice [5], our study presented here did not observe any significant effect from klotho overexpression on running performance of $\mathrm{mdx}^{4 \mathrm{cv}}$ mice. The varying findings may be due to differences in the dystrophin-null murine models used ( $\mathrm{mdx}$ versus $\mathrm{mdx}^{4 \mathrm{cv}}$ ), or more likely, in the type of activity measured. The previously reported study examined treadmill-running ability in klotho overexpressing mdx mice, which evaluates a fundamentally different running activity than long-term voluntary running activity. It is also important to note that the gender of the mice used to examine forced (treadmill) running performance in klotho overexpressing $\mathrm{mdx}$ mice was not specified [5].

While we did not observe an impact of klotho overexpression in dystrophin-null mice, our findings provide valuable insight into experimental design considerations necessary for running wheel studies. The main take home points from our current study are:

- Female mice have increased running performance over male mice across all treatment groups examined.

- Dystrophin-null, $\mathrm{mdx}^{4 \mathrm{cr}}$ mice have reduced overall running performance compared to wild type mice.

- In both females and males, klotho overexpression in $\mathrm{mdx}^{4 \mathrm{cv}}$ mice does not alleviate their reduced running performance and running profiles remains similar with and without klothooverexpression.

- While differences exist between $\mathrm{mdx}^{4 \mathrm{cv}}$ and wild type mice there is no difference in running speed between male and female mice within these strains.

- Male mice perform less active running intervals and are also active for an overall lower percentage of time.
- While there is no difference between $\mathrm{mdx}^{4 \mathrm{cv}}$ and wild type mice in the number of active running intervals, female $\mathrm{mdx}^{4 \mathrm{cv}}$ mice are active for a significantly less percentage of time than wild type female mice.

- Male $\mathrm{mdx}^{4 \mathrm{cr}}$ mice run for shorter intervals than female $\mathrm{mdx}^{4 \mathrm{cv}}$ mice, but wild type males and females do not differ in the length of running intervals.

- $\mathrm{Mdx}^{4 \mathrm{cr}}$ mice rest for a longer length of time between running intervals than wild type mice.

Although previous studies have established in wild type mice that females maintain a greater exercise capacity than males $[32,33]$, running studies in dystrophin-null mice have been typically based on single gender, pooling males and females together, or in some studies gender was not described [11]. For example, in a study demonstrating that mdx mice consistently ran $25 \%$ less distance than the wild type controls, both male and females were pooled together per the studied groups [34]. Our findings clearly establish that gender pooling into one experimental group can complicate the interpretation of the results. The underlying cause of our observed decrease in running performance in male mice is unclear. While it could reflect changes in muscle strength, it is possible that behavioral distinctions between the genders [35] play a role in the reduced persistence in voluntary running in the male mice.

In all, our study provides valuable insight into gender distinction in exercise running, indicating the importance of understanding the natural differences between genders. Our work highlights key considerations that should be accounted for when examining physical activity in mice, particularly for studies involving dystrophin-null animals.

\section{ACKNOWLEDGMENTS}

We would like to thank the Nathan Shock Functional Assessment Core in the Department of Comparative Medicine, In Vivo Services Core facilities for providing the equipment for the wheel running studies. This research was supported by grants from the National Institutes of Health to ZYR (AG021566 and NS090051). MP was a recipient of a postdoctoral fellowship from the Genetic Approaches to Aging training grant (T32 AG000057). 


\section{SUPPLEMENTARY MATERIAL}

The supplementary material is available in the electronic version of this article: https://dx.doi.org/ 10.3233/JND-210703.

\section{REFERENCES}

[1] Zhou L, Lu H. Targeting fibrosis in Duchenne muscular dystrophy. Journal of Neuropathology \& Experimental Neurology. 2010;69:771-6. https://doi.org/10.1097/ NEN.0b013e3181e9a34b.

[2] Shefer G, Rauner G, Yablonka-Reuveni Z, Benayahu D. Reduced satellite cell numbers and myogenic capacity in aging can be alleviated by endurance exercise. PLoS ONE. 2010;5:e13307. https://doi.org/10.1371/ journal.pone.0013307.

[3] Satoh M, Nagasu H, Morita Y, Yamaguchi TP, Kanwar YS, Kashihara N. Klotho protects against mouse renal fibrosis by inhibiting Wnt signaling. American Journal of Physiology-Renal Physiology. 2012;303:F1641-51. https://doi.org/10.1152/ajprenal.00460.2012.

[4] Sahu A, Mamiya H, Shinde SN, Cheikhi A, Winter LL, Vo NV, et al. Age-related declines in $\alpha$-Klotho drive progenitor cell mitochondrial dysfunction and impaired muscle regeneration. Nature Communications. 2018;9:4859. https://doi.org/10.1038/s41467-018-07253-3.

[5] Wehling-Henricks M, Li Z, Lindsey C, Wang Y, Welc $\mathrm{SS}$, Ramos JN, et al. Klotho gene silencing promotes pathology in the mdx mouse model of Duchenne muscular dystrophy. Human Molecular Genetics. 2016;25:2465-82. https://doi.org/10.1093/hmg/ddw111.

[6] Ahrens HE, Huettemeister J, Schmidt M, Kaether C, von Maltzahn J. Klotho expression is a prerequisite for proper muscle stem cell function and regeneration of skeletal muscle. Skeletal Muscle. 2018;8:20. https://doi.org/ 10.1186/s13395-018-0166-x.

[7] Imura A, Iwano A, Tohyama O, Tsuji Y, Nozaki K, Hashimoto $\mathrm{N}$, et al. Secreted Klotho protein in sera and CSF: Implication for post-translational cleavage in release of Klotho protein from cell membrane. FEBS Letters. 2004;565:143-7. https://doi.org/10.1016/j.febslet. 2004.03.090.

[8] Phelps M, Pettan-Brewer C, Ladiges W, YablonkaReuveni Z. Decline in muscle strength and running endurance in klotho deficient C57BL/6 mice. Biogerontology. 2013;14:729-39. https://doi.org/10.1007/s10522-0139447-2.

[9] Bartling B, Al-Robaiy S, Lehnich H, Binder L, Hiebl B, Simm A. Sex-related differences in the wheel-running activity of mice decline with increasing age. Experimental Gerontology. 2017;87:139-47. https://doi.org/10. 1016/j.exger.2016.04.011.

[10] Dupont-Versteegden EE. Exercise and clenbuterol as strategies to decrease the progression of muscular dystrophy in mdx mice. Journal of Applied Physiology. 1996;80:734-41. https://doi.org/10.1152/jappl.1996.80.3.734.

[11] Dupont-Versteegden EE, McCarter RJ, Katz MS. Voluntary exercise decreases progression of muscular dystrophy in diaphragm of $\mathrm{mdx}$ mice. Journal of Applied Physiology. 1994;77:1736-41. https://doi.org/10.1152/jappl. 1994.77.4.1736.
[12] Lang JM, Esser KA, Dupont-Versteegden EE. Altered activity of signaling pathways in diaphragm and tibialis anterior muscle of dystrophic mice. Exp Biol Med (Maywood). 2004;229:503-11. https://doi.org/10.1177/153 537020422900608 .

[13] Rezende EL, Kelly SA, Gomes FR, Chappell MA, Garland Jr. T. Effects of size, sex, and voluntary running speeds on costs of locomotion in lines of laboratory mice selectively bred for high wheel-running activity. Physiological and Biochemical Zoology. 2006;79:83-99. https://doi.org/10.1086/498187.

[14] Grounds MD, Radley HG, Lynch GS, Nagaraju K, De Luca A. Towards developing standard operating procedures for pre-clinical testing in the mdx mouse model of Duchenne muscular dystrophy. Neurobiology of Disease. 2008;31:119. https://doi.org/10.1016/j.nbd.2008.03.008.

[15] Spangenburg EE, Geiger PC, Leinwand LA, Lowe DA. Regulation of physiological and metabolic function of muscle by female sex steroids. Medicine \& Science in Sports \& Exercise. 2012;44:1653-62. https://doi.org/10. 1249/MSS.0b013e31825871fa.

[16] Hara H, Nolan PM, Scott MO, Bucan M, Wakayama Y, Fischbeck KH. Running endurance abnormality in mdx mice. Muscle \& Nerve. 2002;25:207-11. https://doi. org/10.1002/mus.10023.

[17] Hyzewicz J, Ruegg UT, Takeda S. Comparison of experimental protocols of physical exercise for $\mathrm{mdx}$ mice and Duchenne muscular dystrophy patients. Journal of Neuromuscular Diseases. 2015;2:325-42. https://doi.org/ 10.3233/JND-150106.

[18] Kvedaras M, Minderis P, Fokin A, Ratkevicius A, Venckunas T, Lionikas A. Forced running endurance is influenced by gene(s) on mouse chromosome 10. Front Physiol. 2017;8. https://doi.org/10.3389/fphys.2017.00009.

[19] Call JA, Voelker KA, Wolff AV, McMillan RP, Evans NP, Hulver MW, et al. Endurance capacity in maturing $\mathrm{mdx}$ mice is markedly enhanced by combined voluntary wheel running and green tea extract. Journal of Applied Physiology. 2008;105:923-32. https://doi.org/10.1152/japplphy siol.00028.2008.

[20] Chapman VM, Miller DR, Armstrong D, Caskey CT. Recovery of induced mutations for X chromosome-linked muscular dystrophy in mice. PNAS. 1989;86:1292-6. https://doi.org/10.1073/pnas.86.4.1292.

[21] Danko I, Chapman V, Wolff JA. The Frequency of Revertants in mdx mouse genetic models for Duchenne muscular dystrophy. Pediatr Res. 1992;32:128-31. https:// doi.org/10.1203/00006450-199207000-00025.

[22] Im WB, Phelps SF, Copen EH, Adams EG, Slightom JL, Chamberlain JS. Differential expression of dystrophin isoforms in strains of $\mathrm{mdx}$ mice with different mutations. Human Molecular Genetics. 1996;5:1149-53. https://doi.org/10.1093/hmg/5.8.1149.

[23] Banks GB, Combs AC, Chamberlain JS. Sequencing protocols to genotype $\mathrm{mdx}, \mathrm{mdx} 4 \mathrm{cv}$, and $\mathrm{mdx} 5 \mathrm{cv}$ mice. Muscle \& Nerve. 2010;42:268-70. https://doi.org/10. 1002/mus.21700.

[24] Dowling P, Gargan S, Zweyer M, Henry M, Meleady $\mathrm{P}$, Swandulla D, et al. Proteome-wide changes in the mdx-4cv spleen due to pathophysiological cross talk with dystrophin-deficient skeletal muscle. IScience. 2020; 23:101500. https://doi.org/10.1016/j.isci.2020.101500.

[25] Ramos JN, Hollinger K, Bengtsson NE, Allen JM, Hauschka SD, Chamberlain JS. Development of novel micro-dystrophins with enhanced functionality. 
Molecular Therapy. 2019;27:623-35. https://doi.org/10. 1016/j.ymthe.2019.01.002.

[26] Siepka SM, Takahashi JS. Methods to record circadian rhythm wheel running activity in mice. In: Young MW, editor. Methods in Enzymology, vol. 393, Academic Press; 2005, pp. 230-9. https://doi.org/10.1016/S00766879(05)93008-5.

[27] Bernheim J, Benchetrit S. The potential roles of FGF23 and Klotho in the prognosis of renal and cardiovascular diseases. Nephrol Dial Transplant. 2011;26:2433-8. https:// doi.org/10.1093/ndt/gfr208.

[28] Dubal DB, Yokoyama JS, Zhu L, Broestl L, Worden K, Wang D, et al. Life extension factor klotho enhances cognition. Cell Reports. 2014;7:1065-76. https:// doi.org/10.1016/j.celrep.2014.03.076.

[29] Xie J, Cha S-K, An S-W, Kuro-o M, Birnbaumer L, Huang C-L. Cardioprotection by Klotho through downregulation of TRPC6 channels in the mouse heart. Nature Communications. 2012;3:1238. https://doi.org/10.1038/ncomms2240.

[30] Imura A, Tsuji Y, Murata M, Maeda R, Kubota K, Iwano A, et al. $\alpha$-Klotho as a regulator of calcium homeostasis. Science. 2007;316:1615-8. https://doi.org/10.1126/ science.1135901.
[31] Kurosu H, Yamamoto M, Clark JD, Pastor JV, Nandi A, Gurnani P, et al. Suppression of aging in mice by the hormone klotho. Science. 2005;309:1829-33. https:// doi.org/10.1126/science.1112766.

[32] Koteja P, Swallow JG, Carter PA, Garland Jr Theodore. Energy cost of wheel running in house mice: Implications for coadaptation of locomotion and energy budgets. Physiological and Biochemical Zoology. 1999;72:238-49. https://doi.org/10.1086/316653.

[33] Oydanich M, Babici D, Zhang J, Rynecki N, Vatner DE, Vatner SF. Mechanisms of sex differences in exercise capacity. American Journal of Physiology-Regulatory, Integrative and Comparative Physiology. 2019;316:R832-8. https://doi.org/10.1152/ajpregu.00394.2018.

[34] Landisch RM, Kosir AM, Nelson SA, Baltgalvis KA, Lowe DA. Adaptive and nonadaptive responses to voluntary wheel running by mdx mice. Muscle \& Nerve. 2008;38:1290-3. https://doi.org/10.1002/mus.21141.

[35] Balart P, Oosterveen M. Females show more sustained performance during test-taking than males. Nature Communications. 2019;10:3798. https://doi.org/10.1038/s41467019-11691-y. 